\title{
LOS PRIMEROS CATÁLOGOS DE FUENTES TERMALES EN COSTA RICA REALIZADOS POR EL NATURALISTA ALEMÁN ALEXANDER VON FRANTZIUS EN 1862 Y 1873
}

\author{
Asdrúbal G. Vargas ${ }^{1 *} \&$ Guillermo E. Alvarado² \\ C. S. Exploración Subterránea, Área de Perforación e Inyección \\ Área de Amenazas y Auscultación Sísmica y Volcánica (A3SV), \\ Instituto Costarricense de Electricidad (ICE), Apdo. 10032 -1000 San José, Costa Rica. \\ *Autor para contacto: avargass@ice.go.cr
}

(Recibido: 08/02/07; aceptado: 07/05/07)

\begin{abstract}
Two scientific articles, written by the German naturalist Alexander von Frantzius, in the second part of 19th century, are analyzed. Both contributions expose the geographical and hydrogeochemical features of thermal springs in Costa Rica and discuss the relationship between the thermal springs and geological faulted blocks, intrusive bodies, and volcanoes. The geoscientist von Frantzius presents, by first time in Costa Rica, a catalogue with 28 thermal springs, and the first chemical analyses, temperature and flow data, historical aspects and the possible therapeutic and industrial use. Some of the thermal springs identified by von Frantzius are not reported by similar studies made in the second part of the $20^{\text {th }}$ century, and this situation leads for the advanced investigations.

Key words: Von Frantzius, catalogue, thermal springs, chemical analyses, Costa Rica.

RESUMEN: Se analizan dos artículos científicos escritos por el naturalista alemán Alexander von Frantzius en la segunda mitad del siglo XIX. Ambas contribuciones exponen las características geográficas e hidrogeoquímicas de varias fuentes termales en Costa Rica, y discuten la asociación de las fuentes termales con la existencia de fallas geológicas, la existencia de cuerpos intrusivos y volcanes. Frantzius presenta los primeros catálogos de las fuentes termales (28 en total), así como los primeros análisis químicos, datos de temperatura, estimaciones de caudal, aspectos históricos y su posible aplicabilidad con fines terapéuticos y de uso industrial local. Varias fuentes termales ubicadas por von Frantzius al parecer no están reportadas por los estudios similares realizados durante la segunda mitad del siglo XX, lo cual abre la posibilidad para su búsqueda en un futuro cercano.

Palabras clave: Von Frantzius, catálogo, fuentes termales, análisis químicos, Costa Rica.
\end{abstract}




\section{INTRODUCCIÓN}

El reconocido naturalista alemán Alexander von Frantzius (Fig. 1), quien estuvo en Costa Rica entre 1853 y 1869 , escribió una serie de artículos, siendo los más conocidos por los geólogos, aquellos referidos a los volcanes (v.g., von Frantzius, 1861). Sin embargo, dos de estos artículos (von Frantzius, 1862, 1873), los primeros en su tipo, no eran del conocimiento de la mayoría de geocientíficos. La difusión de dichos artículos en nuestro medio fue muy escueta, debido a que se escribieron en idioma alemán y en revistas de poca distribución en Costa Rica. De hecho, no están citados en ningún trabajo sobre fuentes termales realizado en el siglo XX. En vista de los aportes al conocimiento hidrogeológico de nuestro país, a la hidrología de las fuentes termales y aspectos geoestructurales, es que se consideró de suma necesidad la traducción de dichos artículos al idioma español y sus respectivos comentarios. Las traducciones acompañan al presente artículo en este número especial.

El objetivo de este trabajo es exponer las consideraciones más relevantes surgidas de la lectura detenida de los artículos publicados por el geocientífico Alexander von Frantzius, denominados Las fuentes termominerales en Costa Rica (Die warmen Mineralquellen in Costa Rica; von Frantzius, 1862, 1873). No solo son poco conocidos en el medio científico local, sino que aportan los primeros datos e informaciones hidrogeoquímicas sobre las fuentes termales. Ambos artículos son los primeros catálogos de fuentes termales en Costa Rica en forma sinóptica, describiéndose las características de veintiocho fuentes termales. Posteriormente, existen documentos científicos que se refieren a las fuentes termales de Costa Rica, hasta más de un siglo después, con los trabajos de Sáenz (1971), Faillace (1973), Sáenz \& Barquero (1983) y Paniagua (2000). Se consideró importante tratar de ubicar las fuentes termales descritas por von Frantzius y tratar de correlacionarlas con las fuentes y nombres conocidos o reportados en los trabajos de la segunda mitad del siglo XX, dado que en ambos trabajos se utilizaron varias hojas topográficas a escala 1: 50000 editadas por el Instituto Geográfico Nacional.

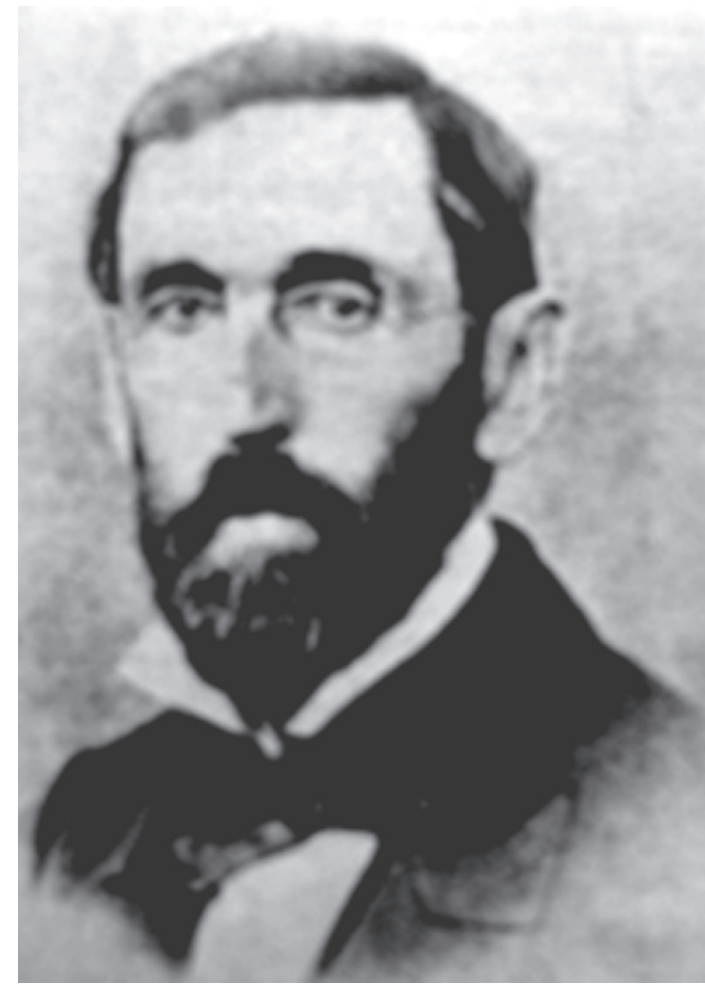

Fig. 1: Alexander von Frantzius (1821-1877).

\section{BIOGRAFÍA DE ALEXANDER VON FRANTZIUS}

Alexander von Frantzius (1821-1877) nació en la ciudad de Danzing, Alemania, el 10 de junio de 1821. En la escuela manifestó gran interés por las ciencias naturales. En 1842 entró en la Universidad de Heidelberg donde permaneció tres años (Tristán, 1942). Estudió medicina en la Universidad de Berlín y se graduó de médico en 1846, aunque más adelante se inclinó por la historia natural. Llegó a Costa Rica en 1853 a sus 32 años, respaldado por cartas de recomendación del presidente de la Academia Imperial de Ciencias Naturales. El motivo de su viaje a estas tierras centroamericanas al parecer no fue único y es, en parte controversial. Tristán (1942) señala, que el motivo del viaje fue la búsqueda de un clima más adecuado para contrarrestar una enfermedad de los pulmones que padecía. Por su parte, Díaz \& Solano (2006) señalan que el motivo fue el gran interés que tenía por las 
ciencias naturales y resolver inquietudes que se formaron en su trayectoria académica y de investigación.

Von Frantzius fue médico en Alajuela y estableció en su residencia un museo con especies de plantas y animales. Además, llevó a cabo observaciones meteorológicas en Alajuela y San José e investigaciones botánicas en la cuenca del río San Juan. Asimismo estudió los volcanes y fuentes termales de Costa Rica, entre otros aspectos, cuyos resultados fueron plasmados en varios artículos que se publicaron en revistas de Alemania en su idioma materno. Realizó prácticas médicas e importantes trabajos en el campo de la historia natural que contribuyeron a aumentar el conocimiento que se tenía de Costa Rica en la ciencia universal (Díaz \& Solano, 2006).

Según Alvarado (2000), con la permanencia de von Frantzius por espacio de 15 años en Costa Rica, se inició una verdadera época de progreso en los conocimientos científicos. En abril de 1859 exploró la cima del volcán Irazú en compañía del ingeniero Francisco Kurtze, efectuando mediciones a alturas barométricas, de temperatura y descripciones sobre la morfología y geología del lugar. En marzo de 1860 exploró el cráter activo del volcán Poás, estimó las dimensiones de la laguna (seis manzanas), analizó las características físicas y químicas del agua, midió por primera vez la temperatura in situ del agua caliente del cráter $\left(39,1^{\circ} \mathrm{C}\right)$, determinó la altura barométrica y describió el ruido y oleaje que se producían a intervalos cuando ascendían grandes burbujas del fondo de la laguna cratérica.

Publicó varios artículos sobre ciencias naturales a partir de información recopilada en Costa Rica. Uno de ellos fue en el año de 1861, titulado en alemán Beiträge zur Kenntniss der Vulkane Costa Rica's (Contribuciones al conocimiento de los volcanes de Costa Rica) en el cual presentó una serie de observaciones realizadas por él en su ascenso a varios volcanes de la cordillera volcánica Central, y que se encuentra traducido al español en el libro Antología del Volcán Poás (Vargas, 1979).

En 1869 partió hacia Washington, dejando enterrada en San José la compañera de su vida que murió victima de una grave enfermedad. De regreso en Alemania, desempeñó la Secretaría de la Asociación Antropológica Alemana hasta el año 1874. Poco después se trasladó a Friburgo, donde murió el 18 de julio de 1877, de su enfermedad pulmonar (Tristán, 1942).

\section{CONSIDERACIONES SOBRE EL ARTÍCULO PUBLICADO EN 1862}

La localización de este artículo se efectuó por medio del Departamento de Bibliotecología de la Escuela Técnica Superior de Aquisgrán (Rheinische Westfallische Technische Hochschule) del estado de Rhenania del Norte, Westfallia, Alemania. "Las fuentes termominerales en Costa Rica" (Die warmen Mineralquellen in Costa Rica), fue publicado en 1862 en una revista de medicina terapéutica, llamada "Revista de medicina prusiana", como un resumen de los resultados más importantes. Sin embargo, esta revista tenía escasa distribución y el mismo Frantzius jamás vio un ejemplar, como él mismo lo señala en una nota al pie de página del artículo de 1873. El artículo que se tradujo consta de 29 párrafos, con un entramado y estructura de las oraciones de tipo técnico, y una gran cantidad de vocablos usados en geología y geografía.

En el artículo se describen veinte fuentes termales agrupadas por situación geográfica. Primero las ubicadas al suroeste de Cartago, las fuentes del valle del río Grande de Candelaria, las fuentes al suroeste de los Montes del Aguacate y finalmente las fuentes del Golfo de Nicoya, Cañas y Liberia. Por causas desconocidas, en este documento no se mencionan las fuentes de Aguacaliente y Orosi de Cartago, ignorándose la razón de dicha omisión, aunque pareciera que el mismo von Frantzius eliminó las primeras cinco fuentes termales o que el editor de la revista, por un error involuntario las omitiera iniciando la numeración de las fuentes con la número 6.

La fuente termal de San Cristóbal, ubicada en el valle del río Grande de Candelaria, es descrita de una manera más amplia que las otras fuentes, pues según von Frantzius, es la fuente más importante por su extensión y caudal, con respecto a todas las fuentes conocidas por él 
en Costa Rica. Considera que podría ser usada como fuente medicinal, aunque no profundiza en este punto.

Incluye datos de temperaturas medidas en el agua de las fuentes, en la unidad de medida Reaumur, que se puede transformar a grados Celsius, por medio de la fórmula $\mathrm{T}\left[{ }^{\circ} \mathrm{C}\right]=1,25 *$ $\mathrm{T}\left[{ }^{\circ} \mathrm{R}\right]$. La fuente termal con la mayor temperatura medida, se encuentra en la margen derecha del brazo oriental del río Barranca, un cuarto de legua (aprox. 1,4 km) aguas arriba del punto donde este brazo se une con el occidental, según la descripción por él indicada. Aunque se incluyen valores de temperatura en las descripciones, las fuentes también las clasifica de manera relativa como fuentes tibias o calientes, tomándose como límite de ambas clasificaciones un valor de temperatura cercano a $24^{\circ} \mathrm{R}\left(30^{\circ} \mathrm{C}\right)$.

La localización de varias fuentes termales descritas en el artículo están referidas a algún río conocido, como por ejemplo Grande de Pirrís, Viejo, Grande de Candelaria, Atarrazú, Parrita, Urén, Sixaola, Virilla, Machuca y Barranca. Dado que von Frantzius no disponía de un sistema geográfico de coordenadas, como se usan actualmente, debió de recurrir a la localización de poblados o ciertas características de los ríos para referenciar espacialmente las fuentes. Estas descripciones crean confusión o dificultan la localización de las fuentes, pues los rasgos morfológicos de algunos ríos han cambiado o el nombre de los poblados ha variado.

Aunque von Frantzius publica los resultados de sus investigaciones en una revista de medicina en Alemania, no se promueven las propiedades curativas terapéuticas de las aguas termales, sino más bien que se dan a conocer las características físico-químicas de las aguas de dichas fuentes de un país poco conocido en Europa como lo era Costa Rica en aquel entonces. Posiblemente, tenía la intención de que otros investigadores vinieran a este país e investigaran más adelante las propiedades curativas de dichas fuentes termales.

En este artículo no se describen las fuentes termales de la vertiente caribe, quizás debido a la falta de caminos y accesos adecuados para llegar a dichas fuentes o a la falta de tiempo y fondos.
Los materiales geológicos que se mencionan en el documento son el pórfiro, masas de carbonato, alumbre y salitre, cristales de sal, sal de cocina, roca caliza y roca intrusiva, depósitos calcáreos y manganeso. El pórfiro es una roca subvolcánica caracterizada por su textura porfídica y generalmente se presenta en forma de diques. El alumbre es el sulfato doble de aluminio y potasio, mientras que el salitre, es considerado como cualquiera de los nitratos minerales que se encuentran en la naturaleza y en especial el nitrato de potasio $\left(\mathrm{KNO}_{3}\right)$. La palabra salitral es una de las más utilizadas en los artículos y se le denomina al lugar donde se acumula salitre y donde llegaban animales a consumir sal formada de manera natural.

Von Frantzius describe además rocas traquíticas (lava alcalina compuesta de feldespato potásico) y las sienitas (su equivalente intrusiva) por su similitud con las rocas europeas. Ambos tipos de rocas son escasas en el territorio nacional según la nomenclatura petrográfica vigente. De hecho ya desde 1836, Leopold von Buch había propuesto el término andesita (lava de los Andes), para aquellas rocas volcánicas exóticas para los europeos, recolectada por las expediciones de Alexander von Humbolt y otros investigadores en los Andes y en Kamchatka, para diferenciarlas de las traquitas europeas.

En varias de las descripciones realizadas directamente en el campo, utilizó básicamente sus sentidos. Así por ejemplo menciona el sabor ligeramente salado de la fuente de San Cristóbal $\left(\mathrm{N}^{\circ}\right.$ 9), mientras que para la $\mathrm{N}^{\circ} 11$, indica que no tenía ningún sabor a sal. En cuanto a la temperatura, señala que para la fuente 15 , el grado de calor es de tal magnitud que la mano se puede mantener en el agua solamente durante un corto tiempo, sugiriendo una temperatura mayor de $45^{\circ} \mathrm{C}$.

\section{Relocalización de las fuentes termales}

Se ha tratado de ubicar espacialmente las fuentes termales descritas, considerando la información aportada por von Frantzius (Cuadro 1).

La fuente $\mathrm{N}^{\circ} 6$ corresponde a la ubicada cerca de la localidad de Coris de Cartago, y está relacionada posiblemente con la falla denominada Guarco 
Cuadro 1

Identificación de las fuentes termales señaladas por v. Frantzius

\begin{tabular}{|c|c|c|c|c|}
\hline $\begin{array}{l}\text { v. Frantzius } \\
1862 \\
\end{array}$ & $\begin{array}{c}\text { v. Frantzius } \\
1873 \\
\end{array}$ & Ubicación. Siglo XIX & Posible correlación S. XX & Referencias \\
\hline $\mathrm{N}^{\circ} 6$ & $\mathrm{~N}^{\circ} 9$ & Sabana Grande de Corio & $\begin{array}{l}\text { Hoja Istarú 3345IV. Lo- } \\
\text { calidad de Bermejo. En la } \\
\text { Q. Barahona aparecen ter- } \\
\text { males. Coord. 538,7-204,5 }\end{array}$ & $\begin{array}{c}\text { Sáenz, 1971; Cotter, 1985; } \\
\text { Sáenz \& Barquero, } 1983\end{array}$ \\
\hline$N^{\circ} 7$ & $\mathrm{~N}^{\circ} 10$ & $\begin{array}{l}\text { Tres Ríos. A una milla de } \\
\text { San José, al este, finca de } \\
\text { Manuel Carazo }\end{array}$ & $\begin{array}{l}\text { Hoja Istarú 3345IV. El AyA } \\
\text { capta manantiales denomi- } \\
\text { nados del Padre Carazo }\end{array}$ & No hay \\
\hline $\mathrm{N}^{\circ} 8$ & $\mathrm{~N}^{\circ} 11$ & $\begin{array}{c}\text { Agua caliente y Salitral de } \\
\text { Desamparados }\end{array}$ & $\begin{array}{l}\text { Hoja Abra } 3345 \text { I. Al } \\
\text { sureste de Desamparados } \\
\text { poblado llamado Salitral. } \\
\text { Coord. 207,0-531,0 }\end{array}$ & $\begin{array}{c}\text { Sáenz, 1971; Sáenz \& } \\
\text { Barquero, } 1983 \text { la ubican en } \\
\text { San Antonio de Desampara- } \\
\text { dos a } 2 \mathrm{~km} \text { al norte del sitio } \\
\text { llamado Salitral y al este de } \\
\text { Desamparados. }\end{array}$ \\
\hline $\mathrm{N}^{\circ} 9$ & $\mathrm{~N}^{\circ} 13$ & $\begin{array}{l}\text { Extremo occidental del } \\
\text { poblado San Cristóbal }\end{array}$ & $\begin{array}{c}\text { Hoja Caraigres 3345II. } \\
\text { Entre poblado San Cristóbal } \\
\text { Norte y Sur }\end{array}$ & $\begin{array}{l}\text { Sáenz, 1971; Sáenz \& } \\
\text { Barquero, } 1983\end{array}$ \\
\hline $\mathrm{N}^{\circ} 10$ & $\mathrm{~N}^{\circ} 14$ & $\begin{array}{l}\text { Margen izquierda río } \\
\text { Atarrazú. Lado norte Cerro } \\
\text { Bustamante en Frailes }\end{array}$ & $\begin{array}{c}\text { Hoja Caraigres 3345II. } \\
\text { Lugar llamado Santa Elena. } \\
\text { Coord. 194,5-530,5 }\end{array}$ & Saénz \& Barquero, 1983 \\
\hline $\mathrm{N}^{\circ} 11$ & $\mathrm{~N}^{\circ} 12$ & $\begin{array}{c}\text { Margen derecha río Parrita. } \\
\text { Boca Dota }\end{array}$ & $\begin{array}{l}\text { No existe equipariedad con } \\
\text { alguna fuente }\end{array}$ & No hay \\
\hline $\mathrm{N}^{\circ} 12$ & $\mathrm{~N}^{\circ} 15$ & Salitral del Rayo & $\begin{array}{c}\text { Hoja Caraigres 3345II. } \\
\text { Posiblemente se refiere a } \\
\text { fuentes que aparecen en las } \\
\text { márgenes del río Grande de } \\
\text { Candelaria }\end{array}$ & $\begin{array}{c}\text { Sáenz, 1971; Saénz \& } \\
\text { Barquero, } 1983\end{array}$ \\
\hline $\mathrm{N}^{\circ} 13$ & $\mathrm{~N}^{\circ} 16$ & Aguacaliente del Cangrejal & $\begin{array}{c}\text { Hoja Caraigres 3345II. } \\
\text { Márgenes del río Grande de } \\
\text { Candelaria cerca de Cangre- } \\
\text { jal de Acosta }\end{array}$ & $\begin{array}{c}\text { Sáenz, 1971, Saénz \& } \\
\text { Barquero, } 1983\end{array}$ \\
\hline $\mathrm{N}^{\circ} 14$ & $\mathrm{~N}^{\circ} 17$ & $\begin{array}{c}\text { Cerca de Puriscal, al sur de } \\
\text { San Rafael, en una garganta } \\
\text { del río Viejo }\end{array}$ & $\begin{array}{l}\text { Hoja Río Grande } 3345 \text { IV, } \\
\text { coincide con los nombres de } \\
\text { las localidades actuales }\end{array}$ & No hay \\
\hline $\mathrm{N}^{\circ} 15$ & $\mathrm{~N}^{\circ} 3$ & $\begin{array}{c}\text { A los pies del cerro Dota, a } \\
4 \text { leguas de Térraba. }\end{array}$ & No hay & Sin nombre. No hay. \\
\hline $\mathrm{N}^{\circ} 16$ & $\mathrm{~N}^{\circ} 2$ & $\begin{array}{l}\text { Falda norte del Cerro Pico } \\
\text { Blanco, valle del río Urén }\end{array}$ & Hoja Sixaola & Sin nombre. No hay. \\
\hline $\mathrm{N}^{\circ} 17$ & $\mathrm{~N}^{\circ} 18$ & $\begin{array}{l}\text { Confluencia del río Virilla } \\
\text { con el río Grande }\end{array}$ & $\begin{array}{l}\text { Hoja Río Grande } 3345 \text { IV. } \\
\text { Posible similitud con una } \\
\text { fuente cerca de Balsa de } \\
\text { Atenas. }\end{array}$ & Sáenz \& Barquero, 1983. \\
\hline $\mathrm{N}^{\circ} 18$ & $\mathrm{~N}^{\circ} 21$ & $\begin{array}{l}\text { Paso del Alumbre margen } \\
\text { derecha río Grande entre } \\
\text { San Pablo y Santo Domingo }\end{array}$ & $\begin{array}{l}\text { Hoja Río Grande } 3345 \text { IV. } \\
\text { Bajo Alumbre, quebrada } \\
\text { Los Ángeles y Quebrada } \\
\text { Salitral. Coord. 210,5-484,0 }\end{array}$ & $\begin{array}{l}\text { Sáenz, 1971; Sáenz \& } \\
\text { Barquero, } 1983\end{array}$ \\
\hline
\end{tabular}




\begin{tabular}{|c|c|c|c|c|}
\hline $\mathrm{N}^{\circ} 19$ & $\mathrm{~N}^{\circ} 22$ & $\begin{array}{l}\text { Puerto Caldera, ladera norte } \\
\text { del cerro, orilla del mar. }\end{array}$ & Caldera & Sáenz, 1971 \\
\hline $\mathrm{N}^{\circ} 20$ & $\mathrm{~N}^{\circ} 20$ & $\begin{array}{l}\text { Nacientes del río Machuca } \\
\text { y quebrada Yurro Amarillo }\end{array}$ & $\begin{array}{l}\text { No hay equipariedad con } \\
\text { algún sitio }\end{array}$ & Sin nombre. No Hay \\
\hline $\mathrm{N}^{\circ} 21$ & $\mathrm{~N}^{\circ} 19$ & $\begin{array}{l}\text { Aguacaliente de la Trin- } \\
\text { chera, río Barranca }\end{array}$ & $\begin{array}{c}\text { Hoja Cerca de la planta } \\
\text { Nagatac, } 11 \mathrm{~km} \text { noreste } \\
\text { Esparza }\end{array}$ & $\begin{array}{c}\text { Sáenz, 1971; Sáenz \& } \\
\text { Barquero, } 1983\end{array}$ \\
\hline $\mathrm{N}^{\circ} 22$ & $\mathrm{~N}^{\circ} 26$ & $\begin{array}{l}\text { Entre desembocadura de los } \\
\text { ríos La Palma y Abangares, } \\
\text { al pie de los Cerros Co- } \\
\text { golito (sic.) y Pájaros }\end{array}$ & $\begin{array}{l}\text { No hay equipariedad con } \\
\text { alguna fuente }\end{array}$ & Sin nombre. No hay \\
\hline $\mathrm{N}^{\circ} 23$ & $\mathrm{~N}^{\circ} 25$ & $\begin{array}{l}\text { Al este del poblado Las } \\
\text { Cañas, en las nacientes del } \\
\text { río Abangares }\end{array}$ & $\begin{array}{c}\text { Quebrada La Luz y Río } \\
\text { Aguacaliente }\end{array}$ & $\begin{array}{c}\text { Sáenz, 1971; Sáenz \& } \\
\text { Barquero, } 1983\end{array}$ \\
\hline $\mathrm{N}^{\circ} 24$ & $\mathrm{~N}^{\circ} 27$ & Al noreste de Bagaces & $\begin{array}{c}\text { Hoja Tierras Morenas } 3147 \\
\text { Sitio denominado Salitral. }\end{array}$ & $\begin{array}{l}\text { Sáenz, 1971; Sáenz \& Bar- } \\
\text { quero, 1983; Faillace, } 1973\end{array}$ \\
\hline $\mathrm{N}^{\circ} 25$ & $\mathrm{~N}^{\circ} 28$ & $\begin{array}{l}\text { Al occidente de Liberia, } \\
\text { Hacienda La Cueva }\end{array}$ & $\begin{array}{c}\text { Hoja Ahogados, Corrd. } \\
\text { 296,5-369,0 }\end{array}$ & Sin nombre. No hay \\
\hline No mencionada & $\mathrm{N}^{\circ} 1$ & Río Jurquín (sic.) & Margen río Yorkín & Sáenz \& Barquero, 1983 \\
\hline No mencionada & $\mathrm{N}^{\circ} 4$ & Valle del río Macho & $\begin{array}{c}\text { Hoja Tapantí } 3445 \text { III. Río } \\
\text { Macho. }\end{array}$ & ICE, 1988. \\
\hline No mencionada & $\mathrm{N}^{\circ} 5$ & $\begin{array}{c}\text { Al este del convento de } \\
\text { Orosi. }\end{array}$ & Hoja Tapantí 3445 III & $\begin{array}{c}\text { Sáenz, 1971; Sáenz \& } \\
\text { Barquero, } 1983\end{array}$ \\
\hline No mencionada & $\mathrm{N}^{\circ} 6$ & $\begin{array}{l}\text { Al sur del valle de Orosi, } \\
\text { cerca del convento }\end{array}$ & Hoja Tapantí 3445 III & $\begin{array}{c}\text { Sáenz, 1971; Sáenz \& } \\
\text { Barquero, } 1983\end{array}$ \\
\hline No mencionada & $\mathrm{N}^{\circ} 7$ & $\begin{array}{c}\text { Hacienda Navarro, río } \\
\text { Navarro }\end{array}$ & Hoja Tapantí 3445 III & $\begin{array}{c}\text { Sáenz, 1971; Sáenz \& Bar- } \\
\text { quero 1983; ICE, 1988, }\end{array}$ \\
\hline No mencionada & $\mathrm{N}^{\circ} 8$ & $\begin{array}{l}\text { Margen derecha río Agua- } \\
\text { caliente }\end{array}$ & $\begin{array}{c}\text { Hoja Istarú } 3445 \text { IV, Her- } \\
\text { videro, Río Aguacaliente, } \\
\text { Aguacaliente }\end{array}$ & $\begin{array}{c}\text { Sáenz, 1971; Sáenz \& Bar- } \\
\text { quero, 1983. Mata, } 1999\end{array}$ \\
\hline No mencionada & $\mathrm{N}^{\circ} 23$ & $\begin{array}{l}\text { Nacientes del río Pocosol, } \\
\text { afluente del río San Juan }\end{array}$ & $\begin{array}{c}\text { No hay equivalencia con } \\
\text { alguna fuente }\end{array}$ & Sin nombre. No hay \\
\hline No mencionada & $\mathrm{N}^{\circ} 24$ & $\begin{array}{l}\text { Al sur de las fuentes del río } \\
\text { Pocosol }\end{array}$ & $\begin{array}{c}\text { No hay equivalencia con } \\
\text { alguna fuente }\end{array}$ & Sin nombre. No hay \\
\hline
\end{tabular}

en el mapa sismológico y tectónico de Montero (1993) o Agua Caliente en Montero (2003).

La fuente $\mathrm{N}^{\circ} 7$, corresponde posiblemente con los manantiales del Padre Carazo, captados actualmente por el Instituto Costarricense de Acueductos y Alcantarillados (AyA).

La fuente $\mathrm{N}^{\circ} 8$ está identificada al sur de Desamparados, luego de que se realizaron excavaciones para la construcción de urbanizaciones. Esta fuente era visitada antiguamente por los ciudadanos de San José para la recreación y en menor medida para usos terapéuticos.

La fuente $N^{\circ} 9$, llamada por von Frantzius San Cristóbal y que para él la fuente más importante, es al parecer mencionada posteriormente por otros autores (Sáenz, 1971, Sáenz \& Barquero, 1983, Paniagua, 2000), pero ubicada entre San Cristóbal Norte y San Cristóbal Sur (hoja Caraigres).

La fuente $\mathrm{N}^{\circ} 10$ coincide posiblemente con una fuente termal ubicada en la localidad de Santa Elena, al norte del Cerro Bustamante y cercana a Frailes de Desamparados (hoja Caraigres). Se ubica aguas abajo de la confluencia de los ríos San Lorenzo y el río San Cristóbal Sur y no como señala von Frantzius entre el Grande de Candelaria y el Tarrazú, pues este último fluye al sur del cerro Bustamante. Esta fuente termal probablemente es una manifestación de la presencia de la falla Patio de Agua, descrita por 
Arias \& Denyer (1990a), aunque dichos autores no toman en cuenta este elemento como indicativo de la presencia de dicha falla.

La fuente descrita como No. 12, se puede ubicar en el río Alumbre, afluente del río Santa Elena, en la vertiente norte del cerro Bustamante, $\mathrm{y}$ al oeste de la fuente $\mathrm{N}^{\circ} 10$, sin embargo, no se conserva el nombre de Salitral del Rayo como es mencionado originalmente. Estas fuentes ubicadas en el valle del río Grande de Candelaria, vienen a ser un elemento de reconocimiento de fallamiento, que podría complementar el contexto geoestructural propuesto entre otros por Arias \& Denyer (1991c).

En la margen derecha del río Candelaria se encuentra un sitio denominado Cangrejal (hoja Caraigres, coordenadas $512,5 \mathrm{~N}$ y $194,5 \mathrm{E}$ ), que posiblemente corresponde con la fuente que menciona von Frantzius como Aguacaliente del Cangrejal ( $\left.\mathrm{N}^{\circ} 13\right)$.

De igual modo la fuente No 14, se ubicaría al este de Santiago de Puriscal, cercana al pueblo de San Rafael (hojas Río Grande y Candelaria, extremo sureste), en el río Viejo, afluente del Tabarcia, aunque no hay nombres geográficos que indiquen la presencia directa de dichos manantiales.

La fuente $\mathrm{N}^{\circ} 17$, se ubicaría en la hoja Río Grande. Muy cerca se encuentra la intersección de la falla La Garita con una falla hipotética, según Arias \& Denyer (1990b), aunque no hay descripciones geográficas que destaquen la presencia de dichas fuentes. Es decir, no está reportada en los trabajos subsiguientes del siglo XX, de las fuentes termales de Costa Rica.

La ubicación geográfica de la fuente $\mathrm{N}^{\circ} 18$, es más sencilla de hallar en la hoja topográfica río Grande, ya que continuando río abajo sobre el río Grande, después de la fuente anterior, se llega a un sitio denominado Bajo Alumbre, muy cerca de la confluencia de la quebrada Salitral con el río Grande de Tárcoles. Además, al sur del Bajo Alumbre existe un hito topográfico llamado San Pablo y un cerro con el mismo nombre y al norte una quebrada llamada Santo Domingo, los cuales concuerdan con los nombres de los poblados señalados por von Frantzius.

Con respecto a la fuente $\mathrm{N}^{\circ} .20$, es preciso señalar que la observación detenida del área que comprende las nacientes del río Machuca, ubicada en las hojas Barranca, Río Grande y Naranjo, permite identificar la presencia de varias caleras, es decir sitios donde se explotan depósitos de rocas calcáreas para extraer carbonato de calcio. Estas canteras concuerdan con lo señalado por von Frantzius, es decir a $5,5 \mathrm{~km}$ al norte de San Mateo, en las nacientes del río Machuca y con la presencia de masas calcáreas. Por otro lado, 11 $\mathrm{km}$ al noroeste de San Mateo existe un sitio denominado Salitral y existe también una quebrada con el nombre de Salitre, que dan la idea de la presencia de aguas termales, pero esto ocurre en la cuenca del río Jesús María.

Existe una fuente termal cerca de la planta hidroeléctrica Nagatac (hoja Miramar, coordenadas 226,5-477,5), posiblemente relacionada con la fuente $\mathrm{N}^{\circ} 21$, precisamente a $11 \mathrm{~km}$ al noreste de Esparza, en las márgenes del río Barranca, mencionadas por Sáenz (1971) y Sáenz \& Barquero (1983). Sin embargo, no está claro a qué llama von Frantzius el brazo oriental y brazo occidental del río Barranca, lo cual genera confusión sobre la ubicación precisa de esta fuente. Pese a ello, era conocida ya en tiempos de la colonia española, pues en su artículo de 1873, se menciona que en una crónica de dos misioneros del año 1756, estos indicaban la existencia de un yacimiento de sal, ubicado al norte de Esparza, relacionado posiblemente con una fuente termal. Desgraciadamente, von Frantzius no deja mayores referencias sobre este documento que permitan acceder a estas crónicas.

No hay evidencias geográficas claras sobre la fuentes termales, que según von Frantzius aparecen entre la desembocadura de los ríos La Palma y Abangares $\left(\mathrm{N}^{\circ} 22\right)$. Las únicas fuentes referidas se ubican cerca de Colorado de Abangares (hoja Abangares), citadas por Sáenz, (1971) y Sáenz \& Barquero (1983).

Con respecto a la fuente $\mathrm{N}^{\circ} 23$, según von Frantzius se ubica al este del poblado Las Cañas, en las nacientes del río Abangares. En este sector existen varios nombres que dan la impresión de estar relacionadas con fuentes termales, como el río Aguacaliente, que desemboca en el río Abangares (hoja Juntas), cerca de la localidad de Juntas. Sáenz \& Barquero (1983), mencionan varias fuentes termales dentro de la hoja topográfica Juntas, ubicadas al noroeste de la localidad de Las Juntas, en la quebrada la Luz y en Santa Lucia. 
En referencia a la fuente $\mathrm{N}^{\circ} 24$ situada por von Frantzius en Salitral, existe efectivamente una fuente al noreste de Bagaces, en un sitio denominado así (hoja Tierras Morenas). Sin embargo, la distancia no coincide con la señalada por von Frantzius, pues el sitio se encuentra a $6 \mathrm{~km}$ y el menciona tan solo $1 / 3$ de legua, lo que equivale aproximadamente a 1,7 km. No obstante, en su artículo de 1873 al parecer von Frantzius rectifica al mencionar, que el Von Seebach (1864) encontró un sitio llamado Salitral a 3 leguas (aprox. 15,5 km) de Miravalles, en el camino hacia Bagaces y que existía una fuente termal a $1 / 2$ legua $(2,5 \mathrm{~km})$ al este de este sitio. Esta ubicación sí concuerda con la señalada para las fuentes termales mencionadas, por Faillace (1973), en la compilación de fuentes termales de la provincia de Guanacaste y por Sáenz \& Barquero (1983) y Paniagua (2000).

La fuente termal identificada $\mathrm{N}^{\circ} 25$, situada por von Frantzius en la hacienda la Cueva, podría estar ubicada a $10 \mathrm{~km}$ al noroeste de Liberia (hoja Ahogados, coord. 369-296,5), en la Hacienda la Cueva, no obstante, que no hay nombres geográficos que identifiquen la presencia de dicha fuente y no está identificada en los trabajos sobre fuentes termales del siglo XX.

\section{CONSIDERACIONES SOBRE EL ARTÍCULO PUBLICADO EN 1873}

En 1873, von Frantzius publicó un artículo más completo con el mismo nombre que el de 1862, pero con más datos. Por la cantidad de datos que aporta, los conceptos que expone y el levantamiento geográfico de manantiales que realizó, este artículo es mucho más amplio que el anterior y profundiza en las características físico-químicas de las fuentes y en la esquematización de los ambientes litológicos y tectónicos donde se ubican los manantiales termales. Además, se incluyen las cinco fuentes que en su primer trabajo se excluyen por causas desconocidas y agrega dos adicionales.

Fue publicado en una revista de Mineralogía, Geologíay Paleontologíaalemana(Neues Jahrbuch für Mineralogie, Geologie und Palaeontologie), en Stuttgart. El título de ambos artículos, es el mismo: Die warmen Mineralquellen in Costa Rica. El artículo tiene catorce páginas, consta de 59 párrafos en alemán, con una estructura gramatical de tipo técnico, y gran cantidad de vocablos usados en geología, geoquímica y geografía. La localización de este artículo se efectuó por medio de los departamentos de bibliotecología de la Escuela Técnica Superior de Aquisgrán mencionado, y de la Biblioteca Herzogin Ann Amalia, de la Universidad de Weimar, en Alemania.

Dicho documento consta de dos partes bien definidas. En primer lugar se describen las características generales de las fuentes termales identificadas, su importancia económica, y los principales compuestos químicos que caracterizan dichas fuentes. Posteriormente, se describen veintiocho fuentes termales, separadas por grupos en función de la ubicación espacial, empezando por las fuentes ubicadas al sureste del país a los pies de la cordillera de Talamanca, sureste de Cartago, suroeste de Cartago, sur de San José, suroeste de los Montes del Aguacate, Poco Sol en la vertiente caribe y terminando con las ubicadas en Bagaces, Cañas y Liberia.

Al inicio del documento se menciona la importancia de la crianza de ganado vacuno para la economía de Costa Rica y de la utilidad práctica de la sal en las fuentes termales como alimento para dichos animales. Por lo tanto, se deja entrever la posibilidad de explotar la sal de las fuentes a mayor escala y evitar la importación de dicho producto. La profundidad de la descripción de las fuentes termales es variable. Así por ejemplo, algunas fuentes fueron descritas hasta con un análisis químico, mientras que otras solamente fueron mencionadas, a partir de los datos aportados por informantes.

Se afirma en el artículo que las fuentes termales identificadas podrían ser clasificadas en tres ambientes geológicos: a) asociadas con volcanes activos o dormidos, b) en ambientes de rocas basálticas y traquíticas sobre grandes bloques dislocados (es decir relacionado con bloques fallados) y c) en ambientes de rocas plutónicas. No obstante, lo anterior no se asignan a estos grupos fuentes termales específicas, solamente se indica que las fuentes termales 4 a la 11 se ubican muy cerca de la línea fronteriza entre las cordilleras volcánica y plutónica y que aquellas fuentes con el número 
posterior a la 11 se ubican muy lejos de dicha línea divisoria, ubicándose en un grupo particular.

Von Frantzius considera que las fuentes termales investigadas por él en Costa Rica constituyen una prolongación geológica de las fuentes investigadas por el afamado naturalista y explorador Alexander von Humbolt en Venezuela. No obstante, la evolución tectónica del bloque sur de Mesoamérica ha sido diferente y la existencia de un cinturón de fuentes termales en Venezuela muy probablemente esée relacionado exclusivamente con neotectonismo y no con magmatismo.

Von Frantzius llegó a tener gran conocimiento y comprensión sobre el territorio costarricense. Es así que elaboró varios mapas de Costa Rica, uno de ellos sobre la parte norte (Fig. 2), en el cual se destacan los volcanes y ríos más importantes. Sin embargo, pese a que von Frantzius exploró en gran medida los alrededores del Cerro Dota, no hay detalles topográficos al respecto. Este mapa fue utilizado para llevar a cabo las relocalizaciones de las fuentes termales.

En su artículo se presenta el primer análisis químico en Costa Rica, que corresponde a la fuente termal de Aguacaliente de Cartago, siendo expresadas las unidades de concentración en granos, que es diferente a gramos. No obstante, el mismo autor se lamenta de que no se realizaran más análisis químicos en otras fuentes termales. Se mencionan los compuestos químicos más importantes de las aguas termales de varias fuentes: cloruro de sodio $(\mathrm{NaCl})$, ácido sulfúrico sódico $\left(\mathrm{H}_{2} \mathrm{SO}_{4} \mathrm{Na}\right)$, ácido sulfúrico potásico $\left(\mathrm{H}_{2} \mathrm{SO}_{4} \mathrm{~K}\right)$, ácido sulfúrico ferroso $\left(\mathrm{H}_{2} \mathrm{SO}_{4} \mathrm{Fe}\right)$, ácido sulfúrico cálcico $\left(\mathrm{H}_{2} \mathrm{SO}_{4} \mathrm{Ca}\right)$, ácido carbónico cálcico $\left(\mathrm{H}_{2} \mathrm{CO}_{3} \mathrm{Ca}\right)$ y sílice $\left(\mathrm{SiO}_{2}\right)$.

Una vehemente crítica es lanzada por él a la escasa utilización de las aguas termales por parte de los habitantes. Acota que el aprovechamiento del agua era realizada por dos grupos sociales: los ricos y los pobres. Los pobres se bañaban a cielo abierto sumergiendo ciertas partes del cuerpo dentro del agua termal en los estanques naturales, mientras que los ricos aprovechaban el agua trasladándola hasta las casas de habitación y luego se bañaban en una cubeta o palangana de madera. Por su parte, los indios de Talamanca utilizaban el agua para curar enfermedades de la piel y úlceras. Para ello, el paciente debía ayunar y posteriormente se sumergía la parte afectada en el agua caliente. A diferencia del primer artículo, en este sí se mencionan, aunque no se describen ampliamente, las fuentes termales en el área de Pocosol de San Carlos, en las nacientes del río homónimo, cerca de la frontera con Nicaragua.

\section{Relocalización de las fuentes termales}

La fuente descrita con el $\mathrm{N}^{\circ} 1$, se trata posiblemente de la fuente termal denominada por Saénz \& Barquero (1983) como Tskui, que se encuentra muy cerca de la margen del río Yorkín, en la línea fronteriza entre Costa Rica y Panamá. La fuente $\mathrm{N}^{\circ} 2$ no ha sido posible identificarla. Por su parte la fuente $\mathrm{N}^{\circ} 7$ está identificada en las cercanías de la localidad de Navarro, en la hoja topográfica Tapantí y fueron estudiadas por el ICE (1988). Posiblemente la roca caliza a que se refiere von Frantzius, pertenece a la formación San Miguel.

La fuente denominada $\mathrm{N}^{\circ} 8$, ubicada al sur de Cartago (hoja Istarú), se identifica fácilmente, pues varios nombres geográficos denotan su presencia: Hervidero, río Aguacaliente y la localidad de Aguacaliente (Tabla 1). Esta fuente es conocida desde la época de la colonia española, pues fue usada como fuente de recreo y esparcimiento y, en menor grado, como fuente medicinal. Posteriormente, a $350 \mathrm{~m}$ de estas fuentes fue construida una presa que sería utilizada en la planta hidroeléctrica de Barro Morado (Mata, 1999, p.172). Esta fuente fue mencionada por el viajero alemán Wilhem Marr (Marr, 1853, pág. 383 ), quien señala que tenía una temperatura de $54^{\circ} \mathrm{R}\left(67,5^{\circ} \mathrm{C}\right)$, y que el agua era ferruginosa y con un ligero sabor a ácido carbónico.

Las restantes fuentes termales son las mismas que las anotadas por él en su artículo de 1862, con la salvedad de que el número cambia. Pese a ello, se agregaron dos fuentes más, dentro del grupo de fuentes que quedaron excluidas en el primer artículo, que corresponden con las fuentes de Pocosol de San Carlos. Dichas fuentes deberían estar ubicadas al norte de Muelle de San Carlos o en su defecto corresponderían con las del Volcán Arenal. 


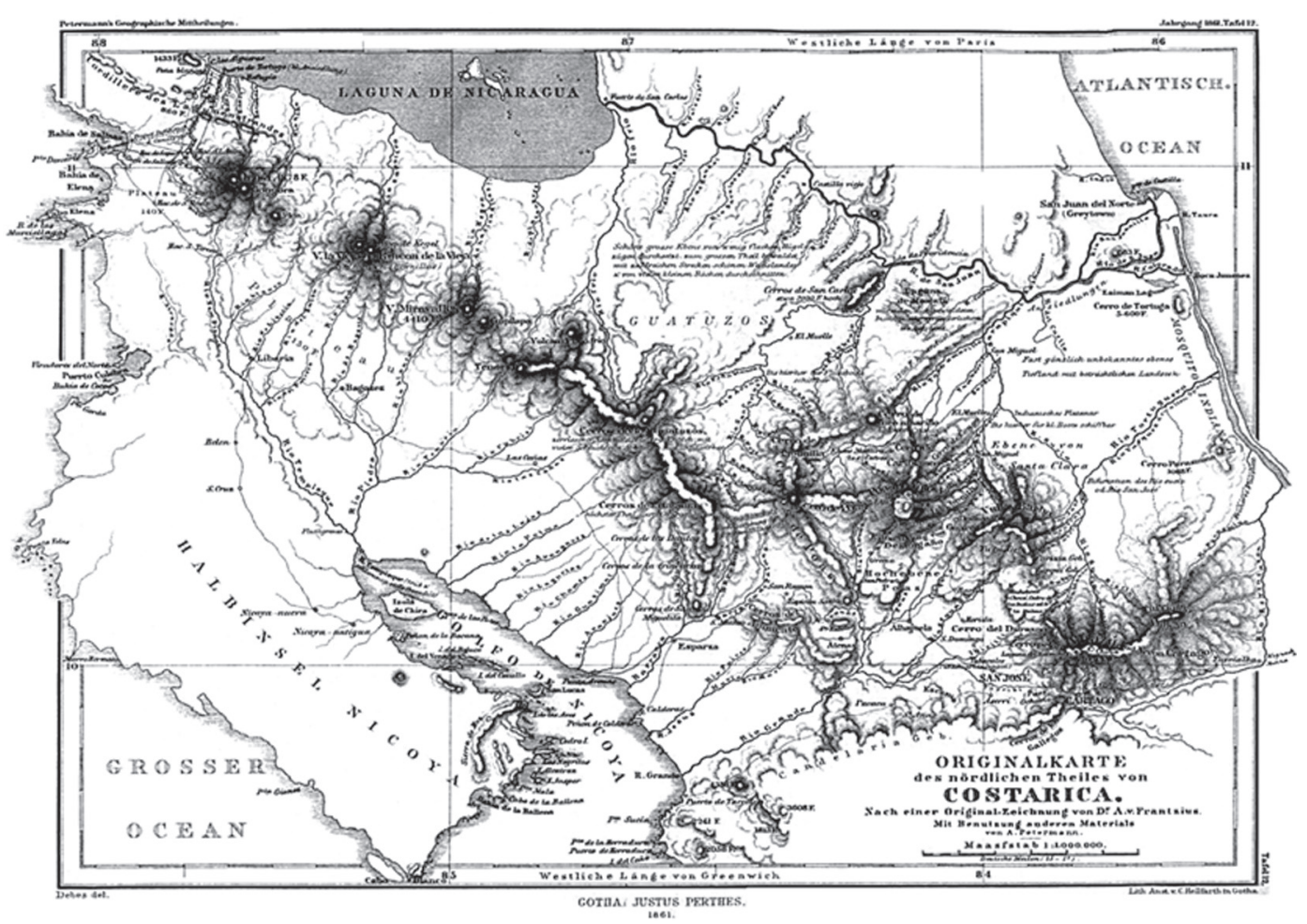

Fig. 2: Mapa geográfico de la parte norte de Costa Rica elaborado por von Frantzius en 1861. Reproducido en A4 al final de este número e incluída en el repositorio de esta artículo.

\section{DISCUSIÓN Y CONCLUSIONES}

Casi un siglo y medio después se trae a la comunidad geocientífica costarricense no solo la traducción de los dos artículos publicados por el afamado naturalista alemán Dr. Alexander von Frantzius en 1862 y en 1873, sino también los comentarios referidos a sus artículos bajo el mismo título "Las fuentes termominerales en Costa Rica" (Die warmen Mineralquellen in Costa Rica).

Dichos trabajos no solo son los primeros dos catálogos de fuentes termominerales en Costa Rica (28 en total), sino que aportan datos hasta ahora desconocidos como el hecho de que al menos 9 fuentes termominerales no están reportadas en ninguno de los trabajos publicados en la segunda mitad del siglo XX. Esto trae una motivación para ir a investigar si todavía existen o si ya desaparecieron como ha pasado con otras fuentes, por ejemplo Salitral en Guanacaste o río Aguacaliente en el volcán Arenal, esta última ahora como fría y potable. De igual modo de las 101 fuentes termales identificadas a la fecha (siglo XXI), un 25\% fueron ya previamente reconocidas por von Frantzius.

De igual interés es el hecho de que ya von Frantzius señalaba tres posibles orígenes válidos aún hoy en día para las fuentes termales: a) asociados con volcanes activos o dormidos; b) en ambientes de rocas basálticas y andesitas sobre grandes bloques fallados y c) en ambientes de rocas intrusivas.

Von Frantzius en su condición de médico y naturalista recalca el hecho de lo poco que eran utilizados dichos recursos naturales para fines terapéuticos y de explotación de sus minerales para la alimentación de la ganadería. No es sino hasta hace escasos 20 años en que se retoman como fuente de entretenimiento y esparcimiento a nivel de todo el territorio nacional, después de que las fuentes termales de Cartago decayeran temporalmente en su popularidad.

Por otro lado, resulta extraño que von Frantzius omitiera las fuentes minerales de Salitral de Santa Ana al sur de San José por su cercanía y la del río Agrio, citada por él en 
1861 (Frantzius, 1861, pág. 24). Ambas fuentes poseen características físico-químicas sobresalientes, y ya para la época de su estadía en Costa Rica existían trochas hacia la comunidad de Santa Ana y Bajos del Toro. Por las descripciones que señala von Frantzius se nota que, para la localización de algunas de las fuentes él utilizó información proveniente de personas que habían visitado las fuentes, por ejemplo las fuentes termales del río Pocosol. De la comparación de las fuentes termales mencionadas por von Frantzius en sus dos artículos con las descritas por autores en la segunda mitad del siglo XX (Cuadro 1), se desprende la conclusión de que el naturalista alemán identificó fuentes que hoy en día no tienen un carácter termal o que desaparecieron en superficie o que simplemente no se han identificado.

\section{AGRADECIMIENTOS}

Agradecemos profundamente a la señora Ursula Heyne Kaussen, bibliotecóloga que labora en la Biblioteca Renana-Westfalliana de la Escuela Técnica Superior de Aquisgrán, por la ayuda brindada para localizar los artículos originales en varias bibliotecas de Alemania. Asimismo queremos expresar el agradecimiento a los revisores que de una u otra manera aportaron comentarios y sugerencias para el mejoramiento de este documento.

\section{REFERENCIAS}

ALVARADO, G.E, 2000: Volcanes de Costa Rica [2ª ed.].- 269 págs. Ed. UNED, San José.

ARIAS, O. \& DENYER, P., 1990a: Geología de la hoja Caraigres.- Escala 1: 50 000. Inst. Geogr. Nac, San José.

ARIAS, O. \& DENYER, P., 1990b: Geología de la hoja Río Grande.- Escala 1: 50 000. Inst. Geogr. Nac, San José

ARIAS, O. \& DENYER, P., 1991c: Estructura geológica de la región comprendida en las hojas topográficas Abra, Caraigres, Candelaria y Río Grande, Costa Rica.Rev. Geol. Amér. Central, 12: 61-74.

COTTER, G., 1985: Aguas minerales de Costa Rica: evaluación y perspectivas.- Tecnología en Marcha. 8(1): 3-9.

DÍAZ, R. \& SOLANO, F., 2006: Las ciencias médicas y su aporte a la institucionalización de la meteorología en Costa Rica (16781936).- Diálogos Revista Electrónica de Historia. 150-183. http://historia.fcs.ucr. ac.cr/dialogos.htm.

FAILLACE, C., 1973: Notas sobre algunas fuentes termominerales en Guanacaste.- Pub. Geol. ICAITI, 4: 47-58.

ICE, 1988: Informe geoquímico de las aguas termales del sureste del Valle Central.48 págs. Instituto Costarricense de Electricidad. Departamento de Geología, San José [Informe interno].

MARR, W., 1853: Viaje a Centroamérica.- 472 págs. Traducido por Reinhold I (2004). Ed. Univ. de Costa Rica, San José.

MATA, J., 1999: Monografía de Cartago.- 802 págs. Ed. Tecnológica de Costa Rica, Cartago.

MONTERO,W., 1993: Mapa sismológico y neotectónico de la gran área metropolitana.- Escala 1: 200 000. Escuela de Geología. UCR. - En: DENYER, P. \& KUSSMAUL, S. (comps.). Atlas geológico Gran Área Metropolitana. Ed. Tecnológica de Costa Rica. Cartago.

MONTERO, 2003: El sistema de falla Atirro-Río Sucio y la cuenca de tracción de TurrialbaIrazú: indentación tectónica relacionada con la colisión del levantamiento del Coco.Rev. Geol. Amér. Central, 28: 5-29.

PANIAGUA, S., 2000: Aguas termales.- En: DENYER, P. \& KUSSMAUL, S. (Eds.): Geología de Costa Rica. Ed. Tecnol. Costa Rica, Cartago: 443-455. 
SÁENZ, R., 1971: Aparatos volcánicos y fuentes termales de Costa Rica.- Dir. Geol., Minas y Petróleo. Inf. Téc. Notas Geol. 41: 1-16.

SÁENZ, R. \& BARQUERO, J., 1983: Fuentes termominerales de Costa Rica.- Bol. Vulcanología, 13: 13-16.

TRISTÁN, J.F., 1942: Extranjeros ilustres en el desenvolvimiento científico de Costa Rica.- Revista de los Archivos Nacionales. 4(1-2): 17-21.
VON FRANTZIUS, A., 1861: Beiträge zur Kenntniss der Vulkane Costa Rica`s. Traducción U. Rehaag. Antología El Volcán Poás. Ed. UNED, San José: 11-33.

VON SEEBACH., K., 1864: Visita a algunos de los volcanes de Guanacaste.- En: MELÉNDEZ, C., 1974: Ministerio de Cultura, Juventud y Deportes, San José: 211-240.

VARGAS, A., 1979: Antología sobre el volcán Poás.- Edit. UNA, San José: 15-25 